\title{
The Influence of Fat Infiltration of Back Extensor Muscles on Osteoporotic Vertebral Fractures
}

\author{
Kwang-Young So, Dae-Hee Kim, Dong-Hyuk Choi, Choong-Young Kim, Jeong-Seok Kim, Yong-Soo Choi \\ Department of Orthopedic Surgery, Kwangju Christian Hospital, Gwangju, Korea
}

\begin{abstract}
Study Design: Retrospective study.
Purpose: To investigate the influence of fat infiltration at low back extensor muscles on osteoporotic vertebral fracture.

Overview of Literature: In persons with stronger back muscles, the risk of osteoporotic vertebral fractures will likely be lower than in those persons with weaker back muscles. However, the degree of influence of fat infiltration of the back extensor muscle on osteoporotic vertebral fracture remains controversial.

Methods: Two hundred and thirty-seven patients who had undergone lumbar spine magnetic resonance imaging and bone mineral density (BMD) were enrolled in this study. The amount of low back extensor muscle was determined using the pseudocoloring technique on an axial view of the L3 level. The patients were divided into two groups: osteoporotic vertebral fracture group (group A) and non-fracture group (group B). The amount of low back extensor muscle is compared with BMD, degenerative change of disc, osteophyte grade of facet joint and promontory angle to reveal the association between these factors.

Results: A negative correlation is found between age and the amount of low back extensor muscle $(p=0.001)$. The amount of low back extensor muscle in group $A$ and group B was $60.3 \% \pm 14.5 \%$ and $64.2 \% \pm 9.3 \%$ respectively, thus showing a significantly smaller amount of low back extensor muscle in the osteoporotic vertebral fracture group $(p=0.015)$.

Conclusions: Fat infiltration of low back extensor muscle was increased in osteoporotic vertebral fracture patients. Therefore, fat infiltration of low back extensor muscle in an elderly person may be a risk factor of osteoporotic vertebral fracture.
\end{abstract}

Keywords: Osteoporotic vertebral fracture; Fat infiltration; Back extensor muscle; Bone mineral density

\section{Introduction}

As the aging population has been rapidly increasing, osteoporosis has been emerging as an important public health issue because it can cause many problems such as fracture, disability, and associated complications which result in significant medical, social, and economic problems and eventually to higher social costs [1,2]. Management of osteoporosis to prevent fracture has become a major issue in public health care, and multi-factorial investigation is currently being carried out. Most of the previous studies have typically focused on the influence of bone mineral density (BMD), bone loss, and mechanical properties that may contribute to vertebral fracture [3-5].

However, Sievanen et al. [6] emphasized the importance of macro-architectural properties as well as microarchitectural properties in vertebral resistance against

Received Dec 27, 2012; Revised Mar 4, 2013; Accepted Mar 4, 2013

Corresponding author: Dong-Hyuk Choi

Department of Orthopedic Surgery, Kwangju Christian Hospital,

37 Yangnim-ro, Nam-gu, Gwangju 503-715, Korea

Tel: +82-62-650-5064, Fax: +82-62-650-5066, E-mail: hjmate@hanmail.net 
fracture. Briggs et al. [7] also mentioned the role of the global environment such as the body position and local environment (including intervertebral disc integrity and paravertebral muscle strength) in preventing vertebral fracture. In 1996, Sinaki et al. [8] suggested that strengthening of back extensor muscle may prevent osteoporotic vertebral fracture and they reported that the relative risk for compression fracture was 2.7 times lower in the backexercise group than in the control group in a prospective 10 year follow-up study [9].

We hypothesized that the fat infiltration of the back extensor muscle could increase the risk of osteoporotic vertebral fracture. Fat infiltration seems to be a late stage of muscular degeneration, and can be measured in a noninvasive manner using magnetic resonance imaging. The aim of this study was to evaluate the influence of fat infiltration of the back extensor muscle on osteoporotic vertebral fracture. We investigated whether the increased fat infiltration of the back extensor muscle had any effect on the development of vertebral compression fracture, or the development of age-related changes such as loss of bone mineral density.

\section{Materials and Methods}

Two hundred and thirty-seven patients who participated in this study underwent both BMD and magnetic resonance imaging (MRI) of the lumbar spine from January 2006 to April 2011. We divided the patients into two groups, depending on the existence of vertebral fracture confirmed by MRI: the fracture group of 96 patients and the non-fracture group of 141 patients. Of the $237 \mathrm{pa}$ tients, 33 were male and 204 were female: 16 male and 80 female in the fracture group and 17 male and 124 female in the non-fracture group. The mean age of the patients was 68.7 years (range, 21-91 years): 70.8 years (range, 50-91 years) in the fracture group and 67.2 years (range, 21-85 years) in the non-fracture group. This study was approved by the Institutional Review Board in Kwangju Christian Hospital.

BMD was obtained with dual-energy X-ray absorptiometry (DXA), and DXA scans were performed and analyzed in accordance with the manufacturer's recommendations (Explorer, Hologic Co., Bedford, MA, USA).

The MR data were acquired on the 1.5-T Signa Excite GE (General Electric, Milwaukee, WI, USA). The subjects were placed in the supine position, with their legs straight and the lumbar spine in a neutral posture. A sagittal localizing sequence was then performed to identify the lumbar disc space intervals. The midpoint of each interspace was used as a reference point and to establish the orientation of the slices for transaxial imaging. After scanning, the images were saved in DICOM file format for Picture Archiving and Communication System and the images were displayed and analyzed using PiView (Infinitt, Seoul, Korea) digital image viewing software. The pseudocoloring technique proposed by Lee et al. [10] was used for measurement of paravertebral muscle mass and fatty infiltration. The T2-weighted axial images of the L3 spine were used. The L3 paravertebral muscles were analyzed because the L3 vertebra was at the center of the lumbar lordotic curvature, so that it could most appropriately reflect the cross sectional area of the paravertebral muscle among the lumbar vertebrae. Moreover, since the osteoporotic vertebral fracture was prevalent in the thoracolumbar junction, the upper lumbar vertebral BMD was frequently disturbed. On the other hand, L4 and L5 are influenced by back muscles such as the multifidus, paraspinal and psoas muscles as these muscles are larger at the lower level, resulting in a significant difference of BMD [10]. Pseudocoloring was chosen as one of the image analyzing tools. Firstly, the regions of interest (ROI) were outlined with a graphic cursor around the back extensor muscle, the fascia thoracolumbalis was then traced down laterally and anteriorly to the dorsal side of the

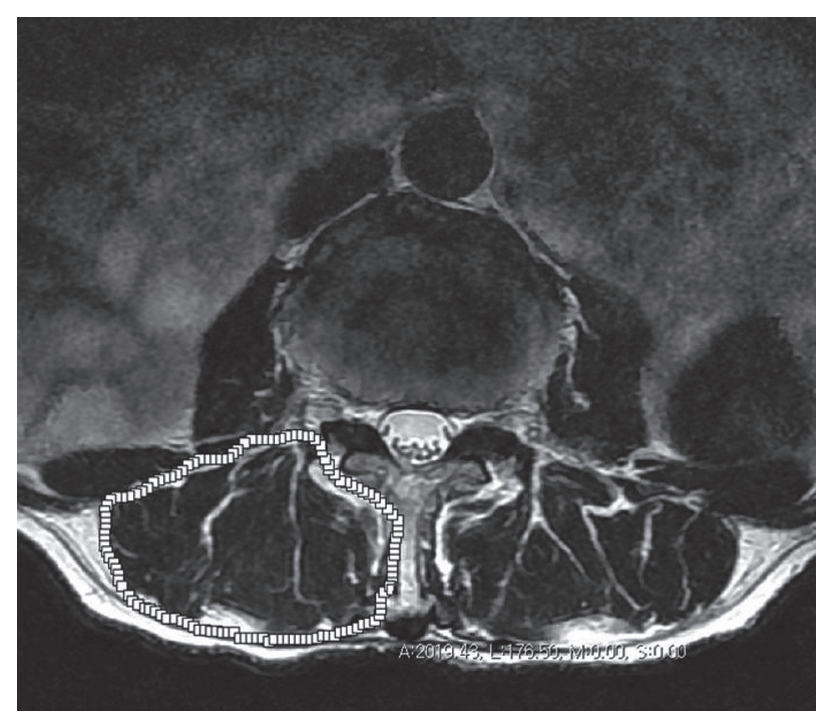

Fig. 1. The T2-weighted axial images at $L 3$ level were analyzed using PiView digital image viewing software. The regions of interest were outlined with a graphic cursor around the back muscle. The fat signal level was determined using a histogram. 
Table 1. Grade of disc degeneration according to Pfirmann grading system and Grade of facet joint osteophyte was classified by Grogan's methods

\section{Disc degeneration}

\begin{tabular}{|ll}
\hline Grade 1 & Homogeneous bright white structure \\
\hline Grade 2 & $\begin{array}{l}\text { Inhomogeneous white structure, possible } \\
\text { horizontal bands }\end{array}$ \\
\hline Grade 3 & $\begin{array}{l}\text { Not white structure but clear distinction } \\
\text { between annulus and nucleus }\end{array}$ \\
\hline Grade 4 & Without clear distinction but not collapsed \\
\hline Grade 5 & Collapsed disc space \\
\hline
\end{tabular}

Facet joint osteophyte

\begin{tabular}{ll} 
Grade 1 & No osteophyte \\
\hline Grade 2 & Possible or small osteophyte \\
\hline Grade 3 & Definite and moderate osteophyte \\
\hline Grade 4 & Large osteophyte \\
\hline
\end{tabular}

quadratus lumborum, followed by the posterior surface of the facet and lamina, and the lateral margin of spinous process (Fig. 1) [10,11]. The fat signal intensity (in gray scale) in the muscle within the ROI was measured using a histogram and the fat signal intensity was applied to the pseudocoloring tool. In this technique, the bright pixels of the fat tissue in the MR images were colored red using the pseudocoloring tool of the program. Subsequently, the percentage of fat infiltrated area in the paravertebral muscle (the percentage of red area in the muscle area) was interpreted (Fig. 2).

The degeneration of the L3-L4 intervertebral disc was evaluated according to the Pfirmann grading system [12], and the grade of osteophyte at the L3-L4 level was classified by Grogan's method (Table 1) [13]. The promontory angle was calculated between the upper margin of the sacrum and the horizontal plane on the lumbar lateral radiograph (Fig. 3).

We analyzed the relationship between the two groups in terms of fat infiltration of the back extensor muscle, age, bone mineral density, the grade of disc degeneration, osteophyte grade at the L3-L4 level, and promontory angle.

Group comparisons for back extensor muscle ratio, disc degeneration at L3-L4, osteophyte at L3-L4, and promontory angle were made using the Student $t$-test. Also, the relationship between fat infiltration of the back extensor muscle and age, body mass index (BMI), disc degeneration, osteophyte, and promontory angle in all the subjects were analyzed using the Spearman correlation analysis. Each result was analyzed using the SPSS ver. 12.0 (SPSS Inc., Chicago, IL, USA) and $p<0.05$ was considered statistically significant.

\section{Results}

The mean lumbar BMD was $0.59 \pm 0.11 \mathrm{~g} / \mathrm{cm}^{2}$ in the fracture group and $0.65 \pm 0.11 \mathrm{~g} / \mathrm{cm}^{2}$ in the non-fracture
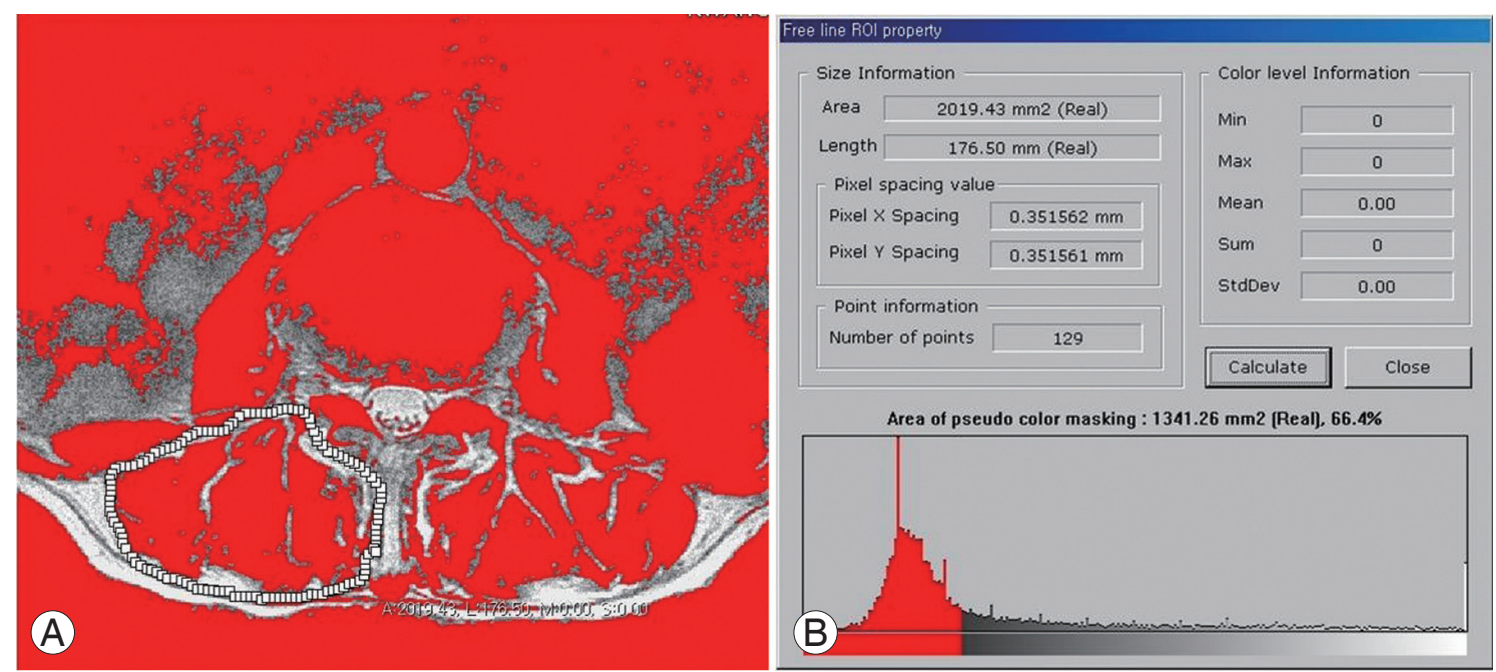

Fig. 2. (A) The pseudocoloring tool of the program was applied on the $L 3$ axial image. The muscle tissue in the magnetic resonance images was colored red. (B) The area of pseudocolor masking of regions of interest was obtained. 
Table 2. Radiologic results between two groups

\begin{tabular}{lccc} 
& Fracture group & Non-fracture group & $p$-value \\
Muscle ratio & $60.3 \pm 14.5$ & $64.2 \pm 9.3$ & 0.015 \\
Disc degeneration & $3.4 \pm 0.6$ & $2.6 \pm 0.7$ & 0.001 \\
Osteophyte & $1.8 \pm 0.7$ & $1.9 \pm 0.5$ & 0.249 \\
Promontory angle & $29.6 \pm 9.7$ & $31.9 \pm 9.0$ & 0.062 \\
\hline
\end{tabular}

Values are mean \pm standard deviation.

Table 3. Relationship between muscle ratio and other results in total patient

\begin{tabular}{lcc} 
& $\begin{array}{c}\text { Correlation with } \\
\text { lumbar muscle ratio }\end{array}$ & $p$-value \\
\hline Age & -0.274 & 0.000 \\
\hline Body mass index & -0.054 & 0.409 \\
\hline Bone mineral density & -0.035 & 0.587 \\
Disc degeneration & 0.016 & 0.811 \\
\hline Osteophyte & 0.080 & 0.219 \\
\hline Promontory angle & 0.055 & 0.395 \\
\hline
\end{tabular}

group. The lumbar BMD was decreased significantly in the fracture group $(p=0.025)$. The mean amount of back extensor muscle ratio was $60.3 \% \pm 14.5 \%$ in the fracture group and $64.2 \% \pm 9.3 \%$ in non-fracture group, and fat infiltration in the back extensor muscle was significantly higher in the fracture group $(p=0.015)$. The mean grade of disc degeneration was $3.4 \pm 0.6$ in the fracture group

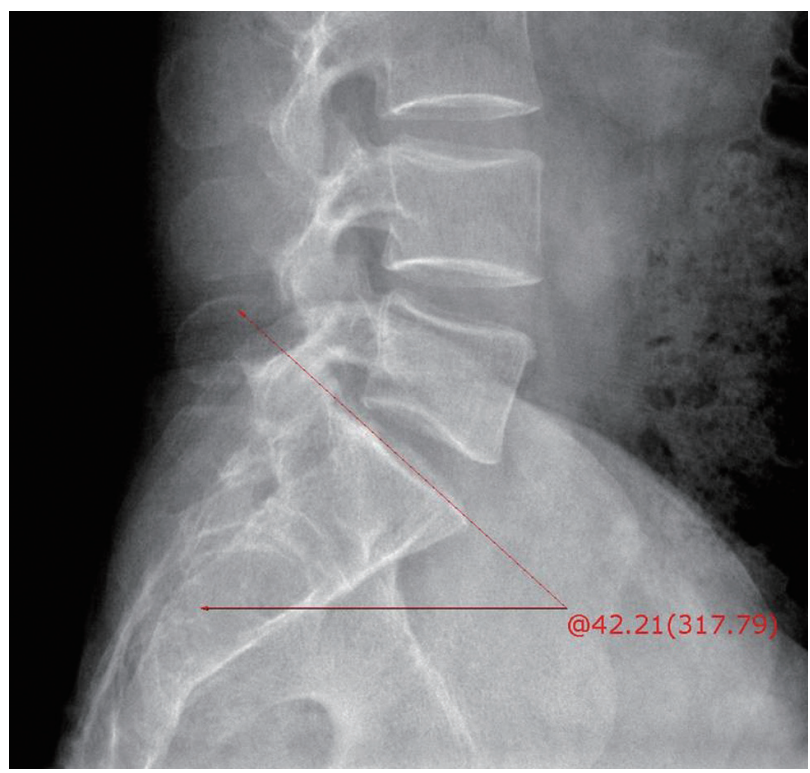

Fig. 3. The promontory angle: the angles between sacral upper margin and horizontal line. and 2.6 \pm 0.7 in the non-fracture group, while the grade of disc degeneration was more progressed in the fracture group ( $p=0.001$ ) (Table 2 ).

In all subjects, the amount of back extensor muscle had a negative correlation with age (Spearman correlation $=-0.247, p=0.001$ ), while other factors such as BMD, BMI, disc degeneration, osteophyte, and promontory angle showed no relationship with the back extensor muscle (Table 3).

\section{Discussion}

The age-related loss of skeletal muscle is characterized by a deterioration of muscle quantity and quality leading to a gradual slowing of movement, a decline in strength and power, and an increased risk of fall-related injuries. Briggs et al. [7] also mentioned the role of the global environment such as body position and local environment (including intervertebral disc integrity and paravertebral muscle strength) in preventing vertebral fracture. Fat infiltration seems to be a late stage of muscular degeneration. However, there was some controversy about the influence of fat infiltration of back muscle on osteoporotic vertebral fracture. We therefore asked whether 1) the fat infiltration of the back extensor muscle could increase the risk of osteoporotic vertebral fracture, 2) aging is related to the fat infiltration of back muscle, and 3) back extensor muscles may affect bone mineral density.

Back muscle may contribute to the compression fracture of vertebrae as a stabilizer and could be a factor affecting BMD. Degeneration of back muscle can weaken the stabilizing force and reduce compression pressure on the vertebral body, which results in a decrease of BMD. We believe there are two factors mainly related to vertebral compression fracture. In this study we therefore determine whether strengthening of the back muscle will improve BMD and lead to a reduction in the incidence of compression fracture. In 1996, Sinaki et al. [8] suggested 
that the strengthening of the back muscle may prevent osteoporotic vertebral fracture and they reported that the relative risk for compression fracture was 2.7 times lower in the back-exercise group than in the control group in a prospective 10 year follow-up study [9]. We then focused on how the back muscle affected the reduction of vertebral compression fracture. The results of this study showed fat infiltration in the back extensor muscle was significantly higher in the fracture group. The reason for the increasing interest in the back extensor muscle is that it is not only considered as a mobilizer but also as a stabilizer for the spine. Without muscular support, the spine has a compression threshold of only $2 \mathrm{~kg}$ before buckling $[13,14]$, which means that paravertebral muscle is an important factor in preventing osteoporotic compression fracture. We believe that dysfunction of back extensor muscles with fat infiltration weakens the stabilizing ability, resulting in greater vulnerability to osteoporotic vertebral fracture. These findings reveal the importance of back extensor strengthening exercises. These results suggest that improvement of back extensor strength could reduce the risk of vertebral fractures later in life.

The degree of fat infiltration with age needs to be specially considered. Sinaki et al. found that back muscle strength was decreased by about $50.4 \%$ in woman aged between 50 to 80 years, with about $2.5 \%$ loss of muscle strength per year. Sinaki et al. [15] mentioned that muscle strength is proportional to the cross sectional area of the extensor muscle. We believe it may be caused by the fatty degeneration of the back extensor muscle with age. A comparison of the cross sectional area can be used as an index to examine the extensor back muscle strength.

Because BMD is known as the most important factor in osteoporotic vertebral fracture, we investigated whether the back extensor muscles affect BMD. To date, several investigators $[16,17]$ have reported that back extensor muscle and the BMD of the spine have a positive correlation, and that strengthening of the back muscle could prevent vertebral fracture. Sinaki et al. [8] also found a negative correlation between the number of vertebral fractures and back muscle strength. However, in a study of the effect of strengthening exercises for back extensor muscles in healthy postmenopausal women, while a significant increase in back extensor muscle strength and improvement of posture was demonstrated, there was no increase in BMD. Also, many previous studies have demonstrated that muscle strengthening exercises increases back muscle strength, but do not increase BMD [18-23]. On the other hand, Halle et al. [24] mentioned that there was a positive relationship between BMD and muscle torque values of the flexor and extensor muscle at the lower lumbar spine in postmenopausal women. However, we could not find any relation between muscle ratio of back extensor and BMD. While the relationship between back extensor muscle and BMD is not clear, we believe that degeneration of back muscle can accelerate the reduction of BMD.

There are some limitations in this study. First, this study is a retrospective study. To obtain a more specific result, a prospective study is needed with a large number of subjects. Also, the subjects were predominantly woman, which means that results are influenced by hormonal effects. Furthermore, since other factors, including hormonal effects, can affect compression fracture, multivariate analysis is needed (although it is not easy to consider all other factors). An additional study to reveal the actual relationship between the amount of paravertebral muscle and back extensor strength is therefore necessary.

\section{Conclusions}

A significantly higher fat infiltration was observed of the back extensor muscle in the osteoporotic vertebral fracture group. The amount of back extensor muscle had a negative correlation with age. Therefore, fat infiltration of back extensor muscle in elderly people is considered a risk factor for osteoporotic vertebral fracture.

\section{Conflict of Interest}

No potential conflict of interest relevant to this article was reported.

\section{References}

1. European Prospective Osteoporosis Study Group, Felsenberg D, Silman AJ, et al. Incidence of vertebral fracture in europe: results from the European Prospective Osteoporosis Study (EPOS). J Bone Miner Res 2002;17:716-24.

2. Oleksik A, Lips P, Dawson A, et al. Health-related quality of life in postmenopausal women with low BMD with or without prevalent vertebral fractures. J Bone Miner Res 2000;15:1384-92. 
3. Hayes WC, Piazza SJ, Zysset PK. Biomechanics of fracture risk prediction of the hip and spine by quantitative computed tomography. Radiol Clin North Am 1991;29:1-18.

4. Hayes WC, Myers ER. Biomechanical considerations of hip and spine fractures in osteoporotic bone. Instr Course Lect 1997;46:431-8.

5. Myers ER, Wilson SE. Biomechanics of osteoporosis and vertebral fracture. Spine (Phila $\mathrm{Pa} 1976$ ) 1997;22:25S-31S.

6. Sievanen H, Kannus P, Nieminen V, Heinonen A, Oja P, Vuori I. Estimation of various mechanical characteristics of human bones using dual energy X-ray absorptiometry: methodology and precision. Bone 1996;18:17S-27S.

7. Briggs AM, Greig AM, Wark JD, Fazzalari NL, Bennell KL. A review of anatomical and mechanical factors affecting vertebral body integrity. Int J Med Sci 2004;1:170-80.

8. Sinaki M, Wollan PC, Scott RW, Gelczer RK. Can strong back extensors prevent vertebral fractures in women with osteoporosis? Mayo Clin Proc 1996;71:951-6.

9. Sinaki M, Itoi E, Wahner HW, et al. Stronger back muscles reduce the incidence of vertebral fractures: a prospective 10 year follow-up of postmenopausal women. Bone 2002;30:836-41.

10. Lee JC, Cha JG, Kim Y, Kim YI, Shin BJ. Quantitative analysis of back muscle degeneration in the patients with the degenerative lumbar flat back using a digital image analysis: comparison with the normal controls. Spine (Phila Pa 1976) 2008;33:318-25.

11. Parkkola R, Rytokoski U, Kormano M. Magnetic resonance imaging of the discs and trunk muscles in patients with chronic low back pain and healthy control subjects. Spine (Phila Pa 1976) 1993;18:830-6.

12. Pfirrmann CW, Metzdorf A, Zanetti M, Hodler J, Boos N. Magnetic resonance classification of lumbar intervertebral disc degeneration. Spine (Phila $\mathrm{Pa}$ 1976) 2001;26:1873-8.

13. Grogan J, Nowicki BH, Schmidt TA, Haughton VM. Lumbar facet joint tropism does not accelerate degeneration of the facet joints. AJNR Am J Neuroradiol 1997; 18:1325-9.
14. Nachemson A. The load on lumbar disks in different positions of the body. Clin Orthop Relat Res 1966;45:107-22.

15. Sinaki M, Nwaogwugwu NC, Phillips BE, Mokri MP. Effect of gender, age, and anthropometry on axial and appendicular muscle strength. Am J Phys Med Rehabil 2001;80:330-8.

16. Sinaki M, McPhee MC, Hodgson SF, Merritt JM, Offord KP. Relationship between bone mineral density of spine and strength of back extensors in healthy postmenopausal women. Mayo Clin Proc 1986;61:116-22.

17. Snow-Harter C, Whalen R, Myburgh K, Arnaud S, Marcus R. Bone mineral density, muscle strength, and recreational exercise in men. J Bone Miner Res 1992;7:1291-6.

18. Bassey EJ, Rothwell MC, Littlewood JJ, Pye DW. Preand postmenopausal women have different bone mineral density responses to the same high-impact exercise. J Bone Miner Res 1998;13:1805-13.

19. Kirk S, Sharp CF, Elbaum N, et al. Effect of longdistance running on bone mass in women. J Bone Miner Res 1989;4:515-22.

20. Kohrt WM, Snead DB, Slatopolsky E, Birge SJ Jr. Additive effects of weight-bearing exercise and estrogen on bone mineral density in older women. J Bone Miner Res 1995;10:1303-11.

21. Pruitt LA, Taaffe DR, Marcus R. Effects of a one-year high-intensity versus low-intensity resistance training program on bone mineral density in older women. J Bone Miner Res 1995;10:1788-95.

22. Sinaki M, Wahner HW, Offord KP, Hodgson SF. Efficacy of nonloading exercises in prevention of vertebral bone loss in postmenopausal women: a controlled trial. Mayo Clin Proc 1989;64:762-9.

23. Gleeson PB, Protas EJ, LeBlanc AD, Schneider VS, Evans HJ. Effects of weight lifting on bone mineral density in premenopausal women. J Bone Miner Res 1990;5:153-8.

24. Halle JS, Smidt GL, O'Dwyer KD, Lin SY. Relationship between trunk muscle torque and bone mineral content of the lumbar spine and hip in healthy postmenopausal women. Phys Ther 1990;70:690-9. 